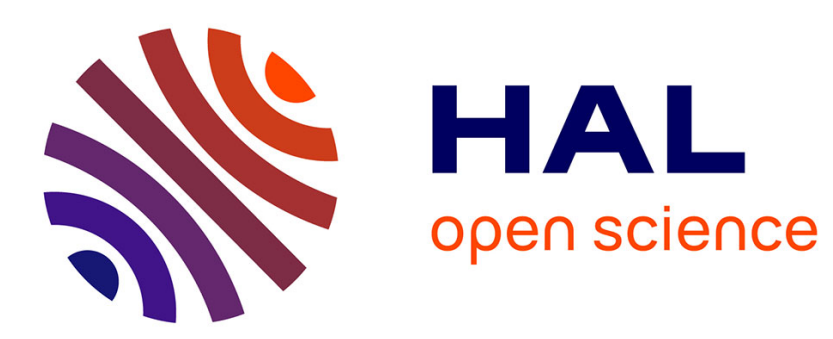

\title{
An improving dynamic programming algorithm to solve the shortest path problem with time windows
}

Nora Touati Moungla, Lucas Létocart, Anass Nagih

\section{To cite this version:}

Nora Touati Moungla, Lucas Létocart, Anass Nagih. An improving dynamic programming algorithm to solve the shortest path problem with time windows. International Symposium on Combinatorial Optimization, Mar 2010, Tunisia. pp.931-938. hal-00522297

\section{HAL Id: hal-00522297 \\ https://hal.science/hal-00522297}

Submitted on 30 Sep 2010

HAL is a multi-disciplinary open access archive for the deposit and dissemination of scientific research documents, whether they are published or not. The documents may come from teaching and research institutions in France or abroad, or from public or private research centers.
L'archive ouverte pluridisciplinaire HAL, est destinée au dépôt et à la diffusion de documents scientifiques de niveau recherche, publiés ou non, émanant des établissements d'enseignement et de recherche français ou étrangers, des laboratoires publics ou privés. 


\title{
An improving dynamic programming algorithm to solve the shortest path problem with time windows
}

\author{
N. Touati Moungla ${ }^{\mathrm{a}}$, L. Létocart ${ }^{\mathrm{b}}, \mathrm{A} \cdot \mathrm{Nagih}^{\mathrm{c}}$ \\ ${ }^{a}$ LIX, École polytechnique, 91128 Palaiseau Cedex, France. \\ ${ }^{b}$ LIPN, UMR 7030 CNRS, Institut Galilée - Université Paris 13, 99 avenue \\ Jean-Baptiste Clément 93430 Villetaneuse, France. \\ ${ }^{c}$ LITA, Université Paul Verlaine, Ile du Saulcy 57045 Metz Cedex 1, France.
}

\begin{abstract}
An efficient use of dynamic programming requires a substantial reduction of the number of labels. We propose in this paper an efficient way of reducing the number of labels saved and dominance computing time. Our approach is validated by experiments on shortest path problem with time windows instances.
\end{abstract}

Keywords: Dynamic programming; Shortest path problem with time windows.

\section{Introduction}

Dynamic Programming (DP) (1) is a well-established method to solve shortest path problems. In the classical shortest path problem, the FordBellman algorithm assigns a single label to each node representing the cost which is gradually improved. An extension of this approach was proposed in (2) in the shortest path problem with ressource constraints context. For the shortest path problem with time windows, the routes must be compared in terms of arrival times at the nodes as well as costs: at each node $i$ a label (arrival time at node $i$, cost of the route) is defined. Several labels have to be stored at node $i$ to calculate the labels of other nodes according to the following optimality principle: If $X_{s j}$ is a minimum cost route from among all the routes from $s$ to $j$ arriving at $j$ at time $T_{j}$ or before, and if $(i, j)$ is its terminal arc, then the sub-route $X_{s i}$ is a minimum cost route from among all routes from $s$ to $i$ arriving at $i$ at time $T_{j}-t_{i j}$ or before, where $t_{i j}$ is the travel time of arc $(i, j)$. 
This procedure can leads to the computation of a large number of labels at each node of the graph. Dominance rules are then used to compare subroutes arriving at a same location for discarding some of them. Dynamic programming algorithms $(2 ; 3)$ rely on labels extension and a dominance procedure (4). In label setting algorithms, nodes are treated once and labels associated to a treated node are kept until the end of the resolution process. In label correcting approaches, nodes are repeatedly treated and their labels extended to all feasible directions.

The dominance procedure applied on each new label requires in the worst case, the exploration of all efficient labels of the current node. Since the number of labels increases exponentialy as a function of the problem's size, dominance computing time increases too. We propose in this work a new variant of the dynamic programming algorithm for accelerating the dominance procedure by reducing the number of labels saved and by identifying efficiently dominated labels.

We present in section 2 the Shortest Path Problem with Time Windows (SPPTW) and describe in section 3 its resolution by the dynamic programming algorithm. We present in section 4 a new variant of this method called dynamic programming with blocs. We validate in section 5 this new approach by experiments on shortest path problem with time windows instances. In section 6 we conclude.

\section{Problem description}

Consider a connected graph $\mathcal{G}=(\mathcal{N}, \mathcal{A})$, where $\mathcal{N}$ is the set of nodes and $\mathcal{A}$ is the set of arcs. With each arc $(i, j) \in \mathcal{A}$ is associated a real number cost $c_{i j}$ and a non negative integer duration $t_{i j}$. Each node $i \in \mathcal{N}$ is characterized by a given time window $\left[a_{i}, b_{i}\right]$ within which the node may be visited. The SPPTW consists in finding the least cost route between two nodes ' $s$ ' and ' $t$ ' in the graph while visiting each node in the specified time window, it can be formulated as follows:

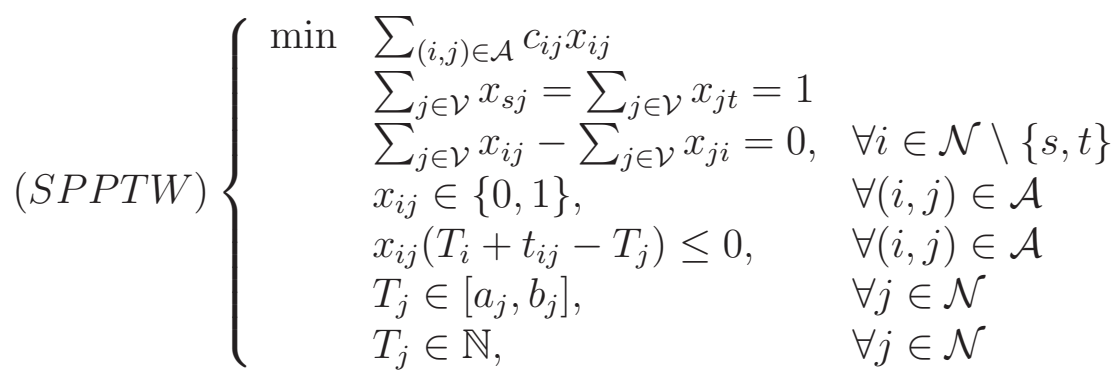


where $x_{i j},(i, j) \in \mathcal{A}$ is the flow binary variable and $T_{i}, i \in \mathcal{N}$ is the visiting time of node $i$.

\section{Dynamic programming algorithm}

We associate to each $l$-th path $X_{s j}^{l}$ from the source $s$ to the node $j$, a (time, cost) label denoted by $E_{j}^{l}=\left(T_{j}^{l}, C_{j}^{l}\right)$, corresponding respectively to the duration and the cost of $X_{s j}^{l}$. This label is computed iteratively along the path $X_{s j}^{l}=\left(v_{0}, v_{1}, \ldots, v_{q}\right)$, where $v_{0}=s$ and $v_{q}=j$ as follows:

$T_{v_{0}}=a_{v_{0}}, C_{v_{0}}=0$

$T_{v_{i}}=\max \left\{a_{v_{i}}, T_{v_{i-1}+t_{v_{i-1} v_{i}}}\right\}, \quad i=1, \ldots, q$

$C_{v_{i}}=C_{v_{i-1}}+c_{v_{i-1} v_{i}}, \quad i=1, \ldots, q$.

A label $E_{j}^{l}$ is feasible iff $T_{v_{i}} \leq b_{v_{i}}, \forall i=1, \ldots, q$. Dominance rules are used to identify and discard useless labels.

Definition 1. Let be $X_{s j}^{1}$ and $X_{s j}^{2}$ two different feasible paths from $s$ to $j$ and $E_{j}^{1}=\left(T_{j}^{1}, C_{j}^{1}\right), E_{j}^{2}=\left(T_{j}^{2}, C_{j}^{2}\right)$ the associated labels respectively. Then, $E_{j}^{1}$ dominates $E_{j}^{2}$ iff $\left(T_{j}^{2}, C_{j}^{2}\right) \geq\left(T_{j}^{1}, C_{j}^{1}\right)$ and $\left(T_{j}^{2}, C_{j}^{2}\right) \neq\left(T_{j}^{1}, C_{j}^{1}\right)$. If $E_{j}^{1}$ is not dominated by another label, it is called efficient.. The complete set of efficient labels characterize the Pareto optimal frontier (Figure 1).

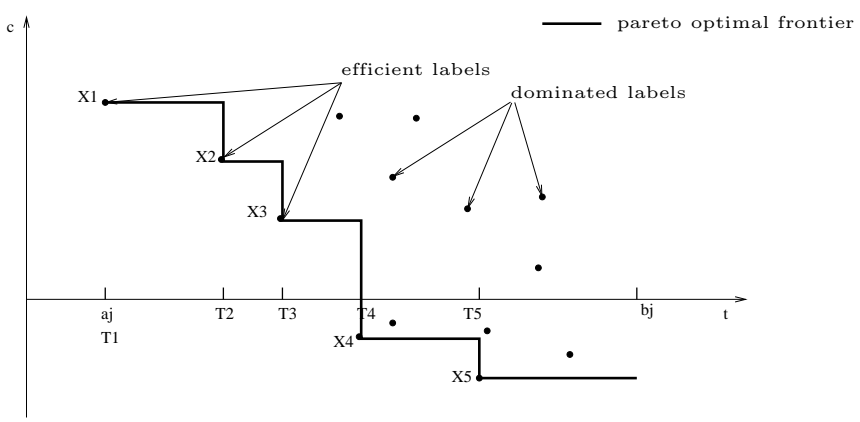

Figure 1: Pareto optimal frontier at node $j$

We denote by $\mathcal{E}_{j}=\left\{E_{j}^{1}, E_{j}^{2}, \ldots, E_{j}^{p}\right\}$ the set of efficient labels computed, and $E_{j}^{p+1}$ a new label at node $j \in \mathcal{N}$. We present on Algorithm 1, the main steps of the dominance procedure applied on $E_{j}^{p+1}$. 


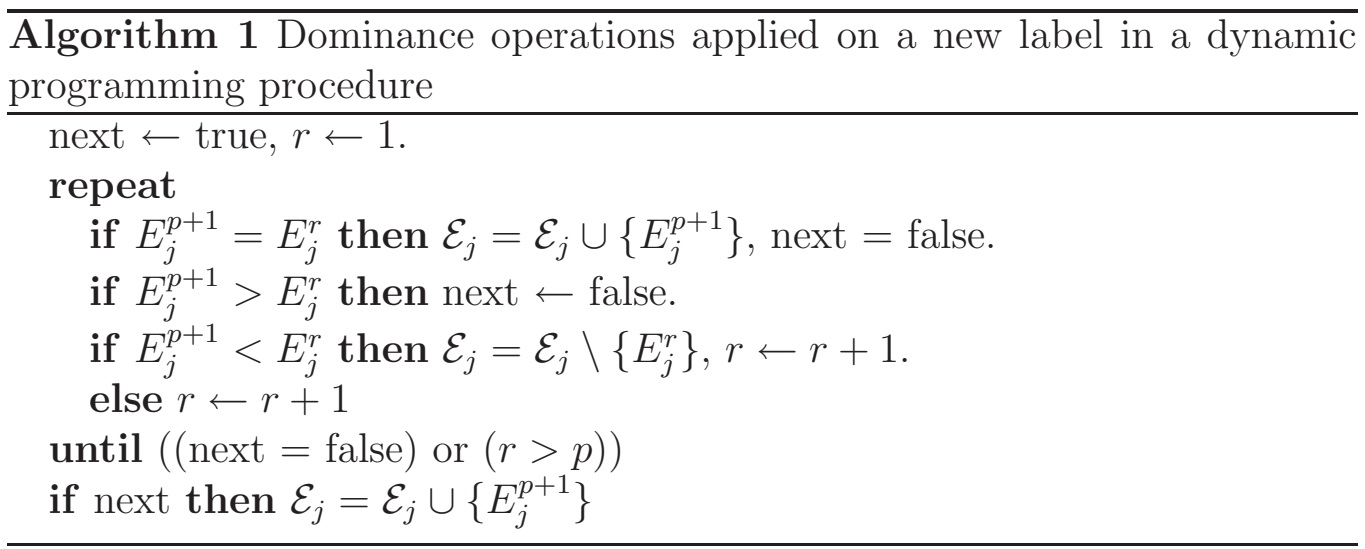

The treatment of a great number of labels increases the computation time of the dominance procedure. We present in the next section an improvement method which the main goal is the reduction of the number of labels saved and the number of labels compared in the dominance procedure.

\section{Dynamic programming with blocs}

The principle of this method is the computation of spaces which contain only efficient labels. We associate to each node $i$ a set of blocs, each bloc is defined by a triplet $B_{i}^{k}=\left(T_{i(l o w)}^{k}, T_{i(u p p)}^{k}, C_{i(u p p)}^{k}\right)$ where $T_{i(\text { low })}^{k}$ corresponds to the lower bound on time, $T_{i(u p p)}^{k}$ define the upper bound on time, and $C_{i(u p p)}^{k}$ represents the upper bound on cost of the bloc indexed by $k$. We suppose that the blocs are not lower bounded by the cost.

\subsection{Initialization}

Let be $i$ the treated node, the set of blocs is initialized by the bloc $B_{i}^{0}=$ $\left(a_{i}, b_{i},+\infty\right)$ (figure 2). The bloc $B_{i}^{0}$ contains only feasible and efficient labels. Let be $E_{i}^{1}=\left(C_{i}^{1}, T_{i}^{1}\right)$ a new label computed at node $i \in \mathcal{N}$ (Figure 3 ). Initially, none efficient label is saved at node $i, E_{i}^{1}$ is then efficient.

The new label $E_{i}^{1}$ allows to characterize the new bloc $B_{i}^{1}=\left(T_{i}^{1}, b_{i}, C_{i}^{1}\right)$ (Figure 3), the bloc $B_{i}^{0}$ is then updated $B_{i}^{0}=\left(a_{i}, T_{i}^{1},+\infty\right)$.

\subsection{Expansion and dominance}

Proposition 1. Let be $E_{i}^{1}, \ldots, E_{i}^{l}$ the set of efficient labels computed at node $i$ and $\mathcal{B}_{i}=\left\{B_{i}^{1}, \ldots, B_{i}^{l}\right\}$ the associated blocs.. We consider $E_{i}=\left(C_{i}, T_{i}\right)$ a new 


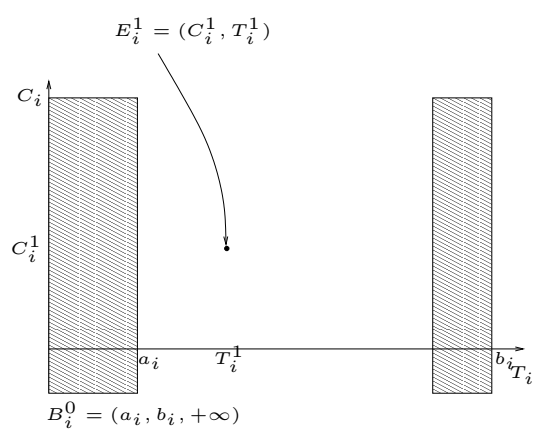

Figure 2: Initialization

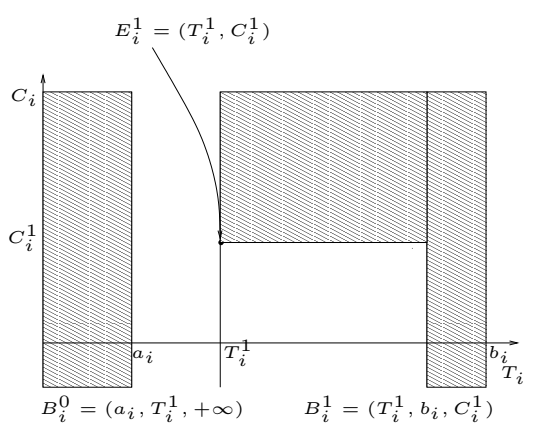

Figure 3: Label expansion

label computed at node $i \in \mathcal{N}$. If the label $E_{i}$ belongs to a bloc $B_{i}^{p}, p=0, \ldots, l$ (i.e.. $T_{i(\text { low })}^{p}<T_{i} \leq T_{i(\text { upp })}^{p}$ and $T_{i}<C_{i(\text { upp })}^{p}$ ), then:

1. $E_{i}$ is a feasible label.

2. $E_{i}$ is an efficient label.

3. The labels dominated by $E_{i}$ are $E_{i}^{p+1}, \ldots, E_{i}^{q}$ which satisfy $C_{i} \leq C_{i}^{p+1}, \ldots, C_{i} \leq$ $C_{i}^{q}$ respectively.

\section{proof 1 .}

1. Each bloc in $\mathcal{B}_{i} \cup B_{i}^{0}$ belongs to the initialized bloc $B_{i}^{0}$ defined in section 4.1. All labels belonging to these blocs are then feasible.

2. (a) If $E_{i}$ belongs to a bloc $B_{i}^{p}, p \in\{1, \ldots, l\}$. Suppose that $E_{i}$ is dominated by the label $E_{i}^{r}, r \in\{1, \ldots, l\}$. Knowing that blocs (labels) are ordered by $T_{l(l o w)}^{k}$ increased, we deduce that the label $E_{i}^{r}$ belongs to a bloc in the set $\left\{B_{i}^{0}, \ldots, B_{i}^{p-1}\right\}$. . On one hand we have $C_{i}^{r} \leq C_{i}$ $\left(E_{i}^{r}\right.$ dominates $\left.E_{i}\right)$ and $C_{i} \leq C_{i}^{p}$ ( $E_{i}$ belongs to the bloc $\left.B_{i}^{p}\right)$, so $C_{i}^{r} \leq C_{i}^{p}$, on another hand $T_{i}^{r} \leq T_{i}^{p}$, hence the contradiction (the labels $E_{i}^{r}$ and $E_{i}^{p}$ are efficient).

(b) If $E_{i}$ belongs to the bloc $B_{i}^{0}$. Knowing that it exists none label in the bloc $B_{i}^{0}$, all efficient labels of node $i$ have durations greater than $T_{i}$, so the label $E_{i}$ is efficient.

3. If $E_{i} \in B_{i}^{p}, p=0, \ldots, l$, then $T_{i} \geq T_{i}^{p-1}>T_{i}^{p-2} \ldots>a_{i}$, we conclude that none label in the set $\left\{E_{i}^{1}, \ldots, E_{i}^{p-1}\right\}$ can be dominated by $E_{i}$. The labels which can be dominated by $E_{i}$ belong to the set $\left\{E_{i}^{p+1}, \ldots, E_{i}^{l}\right\}$, more precisely, the labels dominated by $E_{i}$ are $E_{i}^{p+1}, \ldots, E_{i}^{q}$ where $C_{i} \leq$ $C_{i}^{p+1}, \ldots, C_{i} \leq C_{i}^{q}$ respectively. 


\subsection{An illustrative example}

Figure 4 presents an illustration of the dynamic programming with blocs on an example where $l=6$. Let be $E_{i}^{7}=\left(T_{i}^{7}, C_{i}^{7}\right)$ a new label computed at node $i$. Suppose that $E_{i}^{7}$ belongs to the bloc $B_{i}^{3}$, it is feasible and efficient. Labels dominated by $E_{i}^{7}$ belong to the set $\left\{E_{i}^{4}, E_{i}^{5}, E_{i}^{6}\right\}$ and satisfy $C_{i}^{7} \leq$ $C_{i}^{q}, q \in\{4,5,6\}$, i.e $E_{i}^{4}$. This label and the associated bloc $B_{i}^{4}$ are discarded, the bloc $B_{i}^{7}=\left(T_{i}^{7}, T_{i}^{5}, C_{i}^{7}\right)$ is saved and the bloc $B_{i}^{3}$ is updated $\left(B_{i}^{3}=\right.$ $\left.\left(T_{i}^{3}, T_{i}^{7}, C_{i}^{3}\right)\right)$.
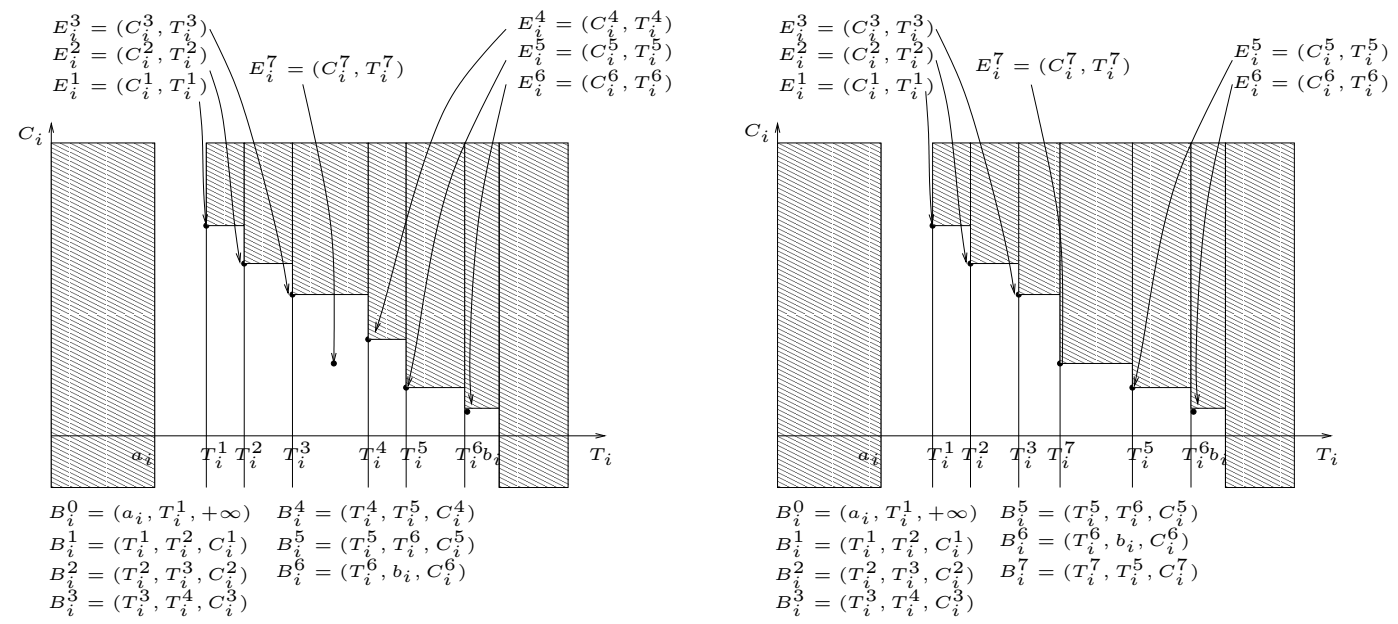

Figure 4: Illustrative example of expansion and dominance procedures

We denote by $\mathcal{B}_{j}=\left\{B_{j}^{0}, E_{j}^{1}, \ldots, E_{j}^{l}\right\}$ the set of blocs computed at node $j \in \mathcal{N}$, and $E_{j}^{p+1}$ a new label at node $j$. We present on Algorithm 2, the main steps of the dominance procedure applied on $E_{j}^{p+1}$ in our new procedure.

\section{Experimentations}

The results reported on Table 1 concern the resolution of the first pricing problem (SPPTW) in a column generation process for the resolution of the vehicle routing problem with time windows on acyclic graphs. The capacity constraints are relaxed. We consider 4 Solomon test instances (C101_25 (25 costumers), $C 101 \_50$ (50 costumers), $C 101$ (100 costumers) and $C 1 \_2 \_1$ (200 costumers)) and 40 randomly generated instances. 10 instances are generated 


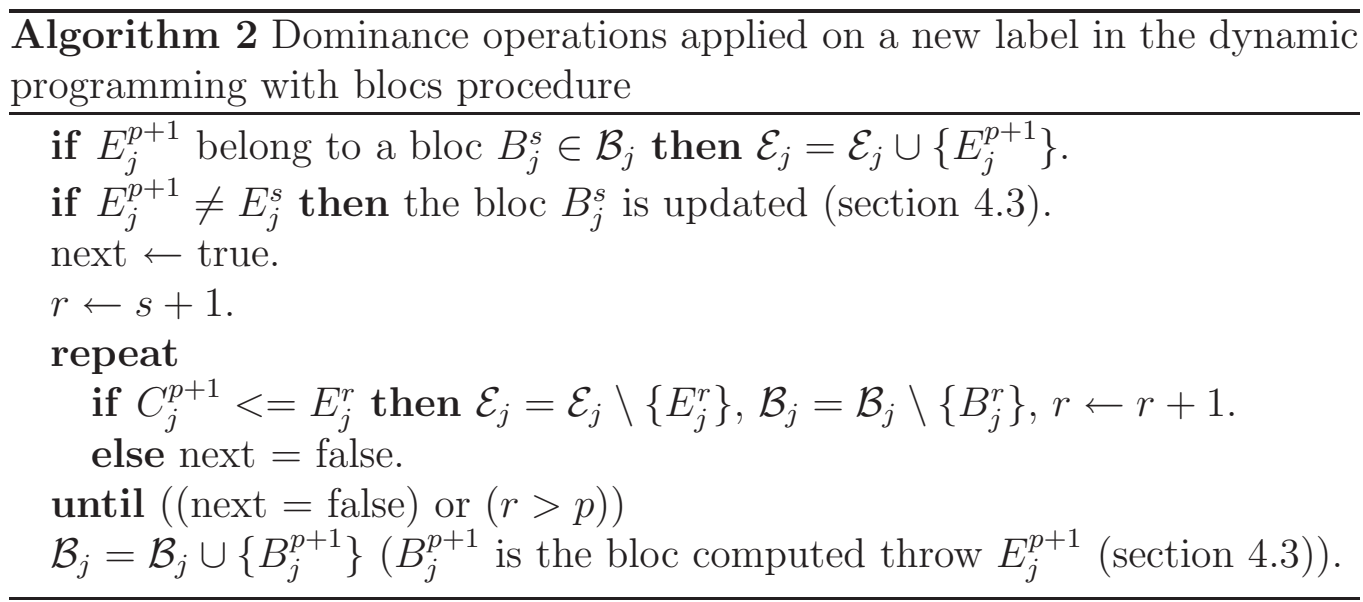

for each size: $G \_100$ (100 costumers), G_120 (120 costumers), G_140 (140 costumers) and $G \_160$ (160 costumers), all results reported for each randomly generated class size are average values over 10 test instances. All instances are solved by the label correcting algorithm 1 .

\begin{tabular}{|c|c|c|c|c|c|c|}
\hline \multirow{2}{*}{$\begin{array}{l}\text { Solomon } \\
\text { instances }\end{array}$} & \multicolumn{3}{|c|}{$C 101 \_25$} & \multicolumn{3}{|c|}{$C 101 \_50$} \\
\hline & resolT & nbLab & nbLabDom & resolT & nbLab & nbLabDom \\
\hline DP_LC & $0,1^{\prime \prime}$ & 343 & 680 & $3,3 "$ & 1673 & 2695 \\
\hline DP_Blocs & $0.04 "$ & 79 & 4 & $0,2 "$ & 204 & 15 \\
\hline \multirow{2}{*}{$\begin{array}{l}\text { Solomon } \\
\text { instances }\end{array}$} & \multicolumn{3}{|c|}{$C 101$} & \multicolumn{3}{|c|}{$C 1 \_2 \_1$} \\
\hline & resolT & nbLab & nbLabDom & resolT & nbLab & nbLabDom \\
\hline DP_LC & $72,0^{\prime \prime}$ & 5941 & 10510 & $2526,0 "$ & 39922 & 1946876 \\
\hline DP_Blocs & $3,2 "$ & 491 & 68 & $87,0 "$ & 1036 & 11425 \\
\hline \multirow{2}{*}{$\begin{array}{l}\text { Generated } \\
\text { instances }\end{array}$} & \multicolumn{3}{|c|}{$G \_100$} & \multicolumn{3}{|c|}{$G \_120$} \\
\hline & resolT & nbLab & nbLabDom & resolT & nbLab & nbLabDom \\
\hline$\overline{D P \_C C}$ & $186,0 "$ & 200586 & 21700018 & $426,0 "$ & 335421 & 116011504 \\
\hline DP_Blocs & $18,9^{\prime \prime}$ & 14446 & 98912 & $24,1 "$ & 92325 & 123413 \\
\hline \multirow{2}{*}{$\begin{array}{l}\text { Generated } \\
\text { instances }\end{array}$} & \multicolumn{3}{|c|}{$G \_140$} & \multicolumn{3}{|c|}{$G \_160$} \\
\hline & resolT & nbLab & nbLabDom & resolT & nbLab & nbLabDom \\
\hline DP_LC & $462,0 "$ & 454729 & 21220354 & $138,0 "$ & 105478 & 11836750 \\
\hline DP_Blocs & $27,0 "$ & 17366 & 94812 & $17,1^{\prime \prime}$ & 57758 & 115377 \\
\hline
\end{tabular}

resolT: resolution time.

nbLab: number of labels treated (labels on which the dominance procedure is applied).

nbLabDom: number of labels compared in the dominance procedure.

Table 1: Dynamic programming with blocs 
Dynamic programming with blocs (DP_Blocs) decrease the number of labels treated by $79 \%$ compared to the label correcting algorithm (DP_LC), this is due to the reduction of the space search of new efficient labels using the blocs. DP_Blocs permits also to reducing efficiently the number of labels compared in the dominance procedure (more than 99\%) compared to DP_LC. These results are predictable, as showed in proposition 2, dominance procedure is applied on a restricted number of labels. These results show the efficiency of the DP_Blocs which permits of decreasing the resolution time of the DP_LC method by $88 \%$.

\section{Conclusion}

Traditionally used dominance rules in dynamic programming consists, after the expansion of a label, to verify if the new label is feasible and not dominated (in the opposite case, it is ignored) by comparing it with all efficient labels already computed. This procedure can be time consuming when the number of labels is large.

We propose in this paper an improving technique which objective is the reduction of the number of labels saved and the computation time of the dominance procedure. In the proposed approach feasibility and dominance tests are included in one, which consists to check if the treated label belongs to a bloc. In the case where the label belongs to a bloc, this method permits a direct access to dominated labels.

Experimentations on SPPTW instances reveal that this method reduces significantly the number of labels saved and the computation time of the dominance procedure.

[1] R. Bellman, Dynamic programming, Princeton university press, NJ, USA, 1957.

[2] M. Desrochers, An algorithm for the shortest path problem with resource constraints, Technical Report G-88-27,GERAD, 1988.

[3] S. Irnich and G. Desaulniers, Shortest path problems with resource constraints, In: Desaulniers et al. (eds), Column generation, Chap. 2. Springer, Berlin, 2005.

[4] M. Desrochers, F. Soumis, 1988. A generalized permanent labelling algorithm for the shortest path problem with time windows. INFOR 3, 191-212. 